\title{
A randomized, controlled study exploring factors associated with decision to undergo HIV screening
}

\author{
Gerard O'Connor', Ailbhe Ni Flaitheartaigh, Aoife Lacey, Jane O'Halloran, Eamon Brazil, Yvette Calderon, \\ Patrick WG Mallon
}

From Abstracts from International Symposium HIV and Emerging Infectious Diseases 2014

Marseille, France. 21-23 May 2013

\section{Introduction}

Little is known of the factors associated with a decision to test for HIV, particularly among varied gender and cultural groups. The Mater-Bronx Rapid HIV Testing (M-BRiHT) project explored if choice of pre-test counselor affected the decision to undergo HIV testing and hypothesized that offering expanded choice of counselor would lead to higher testing completion rates.

\section{Materials and methods}

The M-BRiHT project is a single-site, prospective randomized study of adult Emergency Department attendees offered automated video-based pre-and post-test counseling combined with rapid HIV testing (buccal swab). Subjects were randomly assigned to receive identical, standardized video-based pre- and post-test counseling from a single pre-assigned counselor (Caucasian female) or to choose one of four counselors (male or female from Caucasian or African origin). Primary endpoint was the proportion of subjects completing HIV testing in each randomization group.

\section{Results}

Of 6,000 subjects recruited from September 2012 to 2013, 2950 (49.1\%) were randomized to choice of counselor. Mean (SD) age was 40.9 (16.4) yrs, $48.8 \%$ were female and $91.0 \%, 2.5 \%$ and $3.1 \%$ were of Caucasian, African or Asian ethnicity respectively.

4,919 (82.0\%) completed HIV testing, with significantly higher completion rates in those randomized to choice of counselor (83.1 versus $80.9 \%, \mathrm{P}<0.001)$. Other factors associated with higher HIV test completion rates included younger age (median 36 (SD 15.6) versus 46 $(\mathrm{SD} 18.6)$ yrs, $\mathrm{P}<0.001)$; and male gender $(84.1 \% \mathrm{v}$ $79.8 \%, \mathrm{P}<0.001)$.

Fourteen subjects tested HIV positive (prevalence 2.8/ 1000), with 10 new HIV diagnoses and four re-confirmed HIV positive and re-linked to care. None had symptomatic HIV. Median (IQR) CD4+ T-cell count of new diagnoses was $515(332,595)$ cells/mm3 and all subjects were successfully linked to care.

\section{Conclusions}

The M-BRiHT study demonstrates the feasibility of implementing large scale HIV screening within the Emergency Department and highlights the importance of offering choice of counselor when automated systems are employed to optimize HIV testing rates.

Published: 23 May 2014

doi:10.1186/1471-2334-14-S2-P7

Cite this article as: O'Connor et al:: A randomized, controlled study exploring factors associated with decision to undergo HIV screening. BMC Infectious Diseases 2014 14(Suppl 2):P7. 